\title{
Some Inequalities on Semi-Positive Definite Matrices
}

\author{
Feng Zhang*, Jinli Xu
}

Department of mathematics, Northeast Forestry University, Harbin 150040, China

*Corresponding Author: Feng Zhang, Department of mathematics, Northeast Forestry University, Harbin 150040, China

Abstract: The paper mainly introduces some inequalities of semi-positive definite matrices under partial order relations.

Keywords: Inequalities Lowner partial ordering trace determinant

\section{INTRODUCTION}

Theorem1 1 If $A \geq B \geq 0, A B=B A, f(x)$ is a monotonically increasing function on $(0,+\infty)$, then $f(A) \geq f(B)$.

Proof Because $A, B$ are semi-positive matrices, and $A, B$ can be exchanged, so there is an orthogonal matrix $Q$ for

$$
\begin{aligned}
& A=Q^{t}\left(\begin{array}{llll}
\lambda_{1} & & & \\
& \lambda_{2} & & \\
& & O & \\
& & & \lambda_{n}
\end{array}\right) Q^{\prime B}=Q^{t}\left(\begin{array}{llll}
u_{1} & & & \\
& u_{2} & & \\
& & & \\
& & & u_{n}
\end{array}\right) Q^{\text {, and } \lambda_{i} \geq u_{i}, \forall i \in[1, n] .} \\
& f(A)=Q^{t}\left(\begin{array}{llll}
f\left(\lambda_{1}\right) & & & \\
& f\left(\lambda_{2}\right) & & \\
& & O & \\
& & & f\left(\lambda_{n}\right)
\end{array}\right) Q^{\prime} \quad f(B)=Q^{t}\left(\begin{array}{llll}
f\left(u_{1}\right) & & & \\
& f\left(u_{2}\right) & & \\
& & & f\left(u_{n}\right)
\end{array}\right) Q^{, f(x) \text { is }}
\end{aligned}
$$

a monotonically increasing function on $(0,+\infty)$, so we have $f\left(\lambda_{i}\right) \geq f\left(u_{i}\right), \forall i \in[1, n]$.so $f(A) \geq f(B)$.

With this proposition, we can get the following results:

Proposition 2 We assume $0<A_{k}<I, k=1,2, \ldots, n$, and $A_{i} A_{j}=A_{j} A_{i}$, then $I-\sum_{k} A_{k}<\left(I+\sum_{k} A_{k}\right)^{-1}$.

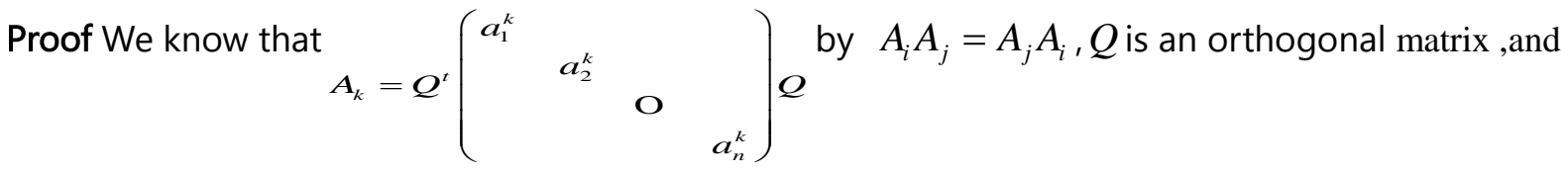
$0<a_{i}^{k}<1$, then<smiles>[Y6][Y9][Y9]</smiles> 
$\left(I+\sum_{k} A_{k}\right)^{-1}=Q^{t}\left(\begin{array}{cccc}\frac{1}{1+\sum_{i} a_{1}^{i}} & & & \\ & \frac{1}{1+\sum_{i} a_{2}^{i}} & & \\ & & & \\ & & & \frac{1}{1+\sum_{i} a_{n}^{i}}\end{array}\right) \quad$, we get $1-\sum_{i} a_{k}^{i}<\frac{1}{1+\sum_{i} a_{k}^{i}}$ by

Weierstrass inequality, so this proposition can be proved.

Corollary 3 We assume that $0<A_{k}<I, k=1,2, \ldots, n$, and $A_{i} A_{j}=A_{j} A_{i}$.

Then $\operatorname{tr}\left(I-\sum_{k} A_{k}\right)<\operatorname{tr}\left(I+\sum_{k} A_{k}\right)^{-1}$

Corollary 4 We suppose that $0<A_{k}<I, k=1,2, \ldots, n$, and $A_{i} A_{j}=A_{j} A_{i}$.

Then $\operatorname{det}\left(I-\sum_{k} A_{k}\right)<\operatorname{det}\left(I+\sum_{k} A_{k}\right)^{-1}$

Proposition 5 We suppose $A_{1} \leq A_{2} \leq \ldots \leq A_{n}$ and $A_{i} A_{j}=A_{j} A_{i}$,

then $\sum_{k}\left(I+A_{k}\right)\left(I+A_{k+1}\right)^{-1} \leq n I+\left(I+A_{1}\right)^{-2}\left(\sum_{k} A_{k}-A_{1}\right)^{2}$,if and only $A_{k}=A_{1}$, the equation holds.

Corollary 6 We consider $A_{1} \leq A_{2} \leq \ldots \leq A_{n}$,

and $A_{i} A_{j}=A_{j} A_{i}$, then $\operatorname{tr} \sum_{k}\left(I+A_{k}\right)\left(I+A_{k+1}\right)^{-1} \leq n+\operatorname{tr}\left[\left(I+A_{1}\right)^{-2}\left(\sum_{k} A_{k}-A_{1}\right)^{2}\right]$

Corollary 7 We assume $A_{1} \leq A_{2} \leq \ldots \leq A_{n}$ and $A_{i} A_{j}=A_{j} A_{i}$,

then $\operatorname{det}\left[\sum_{k}\left(I+A_{k}\right)\left(I+A_{k+1}\right)^{-1}\right] \leq \operatorname{det}\left[n I+\left(I+A_{1}\right)^{-2}\left(\sum_{k} A_{k}-A_{1}\right)^{2}\right]$

Proposition 8 We suppose that $0 \leq a I \leq A_{1} \leq A_{2}, A_{1} A_{2}=A_{2} A_{1}$.

Then $A_{2}^{\frac{1}{n}}-A_{1}^{\frac{1}{n}} \leq\left(A_{2}-a I\right)^{\frac{1}{n}}-\left(A_{1}-a I\right)^{\frac{1}{n}} \leq\left(A_{2}-A_{1}\right)^{\frac{1}{n}}$.

Proposition 9 We suppose $A \geq B \geq 0, A B=B A$, then $\left(A^{p}-B^{p}\right) \leq p(A-B)\left(A^{p-1}+B^{p-1}\right)$, when $1 \leq p<\propto$

Proposition $10 A, B$ are real symmetric positive definite matrices, $A \geq B$ and $A B=B A$, for every $n \in N$,we say

$(A-B)^{2 n+1} \leq 2^{2 n}\left(A^{2 n+1}-B^{2 n+1}\right)$

Proposition $11 \quad c>0$ and $A, B$ are real symmetric positive definite matrices, 
then $\operatorname{tr}(A+B)^{2} \leq \operatorname{tr}\left[(1+c) A^{2}+\left(1+\frac{1}{c}\right) B^{2}\right] \cdot$

Proposition $121 \leq p<\propto, 0<t<1, A, B$ are real symmetric positive definite matrices, and $A B=B A$, then $(A+B)^{p} \leq t^{1-p} A^{p}+(1-t)^{1-p} B^{p}$.

Proposition 13 We suppose $t_{k} \geq 0, \sum_{k=1}^{n} t_{k}=1, A_{1}, \ldots, A_{n}$ are real symmetric positive definite matrices, and $A_{i} A_{j}=A_{j} A_{i}$,when $1 \leq p<\propto$

$\left(\sum_{k=1}^{n} t_{k} A_{k}\right)^{p} \leq\left(\sum_{k=1}^{n} t_{k} A_{k}^{p}\right)$ is established, when $0<p<1$, we $\operatorname{get}\left(\sum_{k=1}^{n} t_{k} A_{k}\right)^{p} \geq\left(\sum_{k=1}^{n} t_{k} A_{k}^{p}\right)$.

Corollary 14 We assume that $t_{k} \geq 0, \sum_{k=1}^{n} t_{k}=1, A_{1}, \ldots ., A_{n}$ are real symmetric positive definite matrices, and $A_{i} A_{j}=A_{j} A_{i}$, when $1 \leq p<\propto$, we have

$\operatorname{tr}\left(\sum_{k=1}^{n} t_{k} A_{k}\right)^{p} \leq \operatorname{tr}\left(\sum_{k=1}^{n} t_{k} A_{k}^{p}\right)$, when $0<p<1$, we get $\operatorname{tr}\left(\sum_{k=1}^{n} t_{k} A_{k}\right)^{p} \geq \operatorname{tr}\left(\sum_{k=1}^{n} t_{k} A_{k}^{p}\right)$.

Corollary 15 We suppose $t_{k} \geq 0, \sum_{k=1}^{n} t_{k}=1, A_{1}, \ldots ., A_{n}$ are real symmetric positive definite matrices, and $A_{i} A_{j}=A_{j} A_{i}$, when $1 \leq p<\propto$, we have

$\operatorname{det}\left(\sum_{k=1}^{n} t_{k} A_{k}\right)^{p} \leq \operatorname{det}\left(\sum_{k=1}^{n} t_{k} A_{k}^{p}\right)$, when $0<p<1,\left(\sum_{k=1}^{n} t_{k} A_{k}\right)^{p} \geq\left(\sum_{k=1}^{n} t_{k} A_{k}^{p}\right)$.

Proposition $16 A_{1}, \ldots, A_{n}$ are real symmetric positive definite matrices, and $A_{i} A_{j}=A_{j} A_{i}$, when $p \geq 1$, we get that

$\sum_{k} A_{k}^{p} \leq\left(\sum_{k} A_{k}\right)^{p} \leq n^{p-1} \sum_{k} A_{k}^{p}$

Proposition17 $0 \leq A \leq I, p>1$, then $(1) \frac{1}{2^{p-1}} I \leq A^{p}+(I-A)^{p} \leq I,(2) A^{p}(I-A)^{p} \leq \frac{1}{4} I$.

Proof When $0<x<1$, we choose a function $f(x)=x^{p}+(1-x)^{p}$, it is not difficult to get $f(x) \geq \frac{1}{2^{p-1}}$, and $f(0)=f(1)=1$, so $f(x) \leq 1$.

By the same way, we can prove $\frac{1}{2^{p-1}} I \leq A^{p}+(I-A)^{p} \leq I$.

Similarly, we get $A^{p}(I-A)^{p} \leq \frac{1}{4} I$.

Proposition $18 A>0$, when $a>1$, we have $(I+A)^{a}>I+a A-\frac{1}{2} a(1-a)\left[A(I+A)^{-1}\right]^{2}$.

Proof We notice that when $x>0$ and $a>1,(I+x)^{a}>I+a x-\frac{1}{2} a(1-a)\left(\frac{x}{1+x}\right)^{2}$ is established. Then we can prove this Proposition.

Corollary $19 A>0, n$ is a positive integer, then 
(1) $\operatorname{tr}(I+A)^{n}>n+\operatorname{tr}\left[n A-\frac{1}{2} n(1-n)\left[A(I+A)^{-1}\right]^{2}\right]$

(2) $\operatorname{det}(I+A)^{n}>\operatorname{det}\left[I+n A-\frac{1}{2} n(1-n)\left[A(I+A)^{-1}\right]^{2}\right]$

Proposition $200<a<1, A+I \geq 0$, then $(I+A)^{a} \geq I+a A[I+(1-a) A]^{-1}$.

Proof when $0<a<1, x \geq-1$, we have $(1+x)^{a} \geq 1+\frac{a x}{1+(1-a) x}$, so this proposition can be proved.

Note when $a>1$ and $-I \leq A \leq \frac{1}{a-1} I,(I+A)^{a} \leq I+a A[I+(1-a) A]^{-1}$.

Proposition $21 A+I \geq 0$, when $0<a<1,(I+A)^{a} \leq I+a A$, when $a<0$ or $a>1$, $(I+A)^{a} \geq I+a A$

Corollary $22 A+I \geq 0$, when $0<a<1, \operatorname{tr}(I+A)^{a} \leq n+\operatorname{atr} A$, when $a<0$ or $a>1$, $\operatorname{tr}(I+A)^{a} \geq n+\operatorname{atr} A$

Corollary $23 A+I \geq 0$, when $0<a<1, \operatorname{det}(I+A)^{a} \leq \operatorname{det}(I+a A)$, when $a<0$ or $a>1$, we have $\operatorname{det}(I+A)^{a} \geq \operatorname{det}(I+a A)$

Corollary $24 A \geq 0$, when $a<0$ or $a>1, \operatorname{det}(I+A)^{a} \geq \operatorname{det}(I+a A) \geq 1+a^{n} \operatorname{det} A$.

Proposition $25 A_{1} \geq A_{2} \geq \ldots . \geq A_{n} \geq 0$ and $A_{i} A_{j}=A_{j} A_{i}, B_{1}, B_{2}, \ldots, B_{n}$ are real symmetric matrices, if $\operatorname{tr} \sum_{i=1}^{k} A_{i} \leq \operatorname{tr} \sum_{i=1}^{k} B_{i}, 1 \leq k \leq n$, then $\operatorname{tr} \sum_{i=1}^{n} A_{i}^{2} \leq \operatorname{tr} \sum_{i=1}^{n} B_{i}^{2}$.

\section{REFERENCES}

[1] Akhmedova V, Zabrodin A. Dispersionless DKP hierarchy and elliptic Löwner equation[J]. Journal of Physics A Mathematical \& Theoretical, 2014, 47(47).

[2] Van Barel M, Vavrin Z. Inversion of a block Löwner matrix[J]. Journal of Computational \& Applied Mathematics, 1996, 69(2):261-284.

[3] Nienhuis B, Kager W. Stochastic Löwner Evolution and the Scaling Limit of Critical Models[J]. Lecture Notes in Physics, 2009, 775:425-467.

[4] Nienhuis B, Kager W. Stochastic Löwner Evolution and the Scaling Limit of Critical Models[M]// Polygons, Polyominoes and Polycubes. 2009.

[5] Rost K, Vavř́n Z. Recursive solution of Löwner-Vandermonde systems of equations. I $己$ [ [J]. Journal of Computational \& Applied Mathematics, 1996, 114(2):319-331.

[6] Uchiyama M. Operator Monotone Functions which Are Defined Implicitly and Operator Inequalities[J]. Journal of Functional Analysis, 2000, 175(2):330-347.

Citation: Feng Zhang \& Jinli Xu, (2019). Some Inequalities on Semi-Positive Definite Matrices. International Journal of Scientific and Innovative Mathematical Research (IJSIMR), 7(4), pp.28-31. http://dx.doi.org/10.20431/2347-3142 .0704005

Copyright: () 2019 Authors, this is an open-access article distributed under the terms of the Creative Commons Attribution License, which permits unrestricted use, distribution, and reproduction in any medium, provided the original author and source are credited. 